\title{
A NOTE ON THE STRONG LAW OF LARGE NUMBERS
}

\author{
BY K. G. BINMORE AND MELVIN KATZ ${ }^{1}$
}

Communicated by David Blackwell, April 3, 1968

1. Introduction. Let $\left\{X_{k}\right\}$ denote a sequence of independent, identically distributed (i.i.d.) random variables. Let

$$
S_{n}=\sum_{k=1}^{n} X_{k} \quad(n=1,2, \cdots) .
$$

A long standing problem in probability theory has been to find necessary and sufficient conditions on the distribution function of $X_{k}$ in order that $n^{-1} S_{n}$ converge almost surely to plus infinity. The purpose of this paper is to exhibit such conditions.

2. TheOREM. Let $\left\{X_{k}\right\}$ denote a sequence of i.i.d. random variables with common characteristic function $\phi$. Then $n^{-1} S_{n} \rightarrow+\infty$ a.s. if and only if

$$
\lim _{b \rightarrow \infty} \int_{-1}^{1} \frac{e^{i u b}-1}{i u} \log \left\{1-\frac{e^{-i u a} \phi(u)}{1+u^{2}}\right\}^{-1} d u
$$

is finite for each $a>0$.

The proof of the theorem is based on the following lemma.

Lemma. Let $\left\{X_{k}\right\}$ denote a sequence of i.i.d. random variables. Then (a) $n^{-1} S_{n} \rightarrow+\infty$ a.s.

if and only if, for each $a>0$,

(b) $\sum_{n=1}^{\infty} n^{-1} P\left(S_{n}<a n\right)<\infty$.

Proof of the Lemma. We first show that (a) implies (b). Suppose there exists an $a>0$ such that $\sum n^{-1} P\left(S_{n}<a n\right)=\infty$. Let

$$
T_{n}=\sum_{k=1}^{n}\left(a-X_{k}\right) \text {. }
$$

Then $\sum n^{-1} P\left(T_{n}>0\right)=\infty$, and, by a theorem of Spitzer [2] it follows that $\lim \sup T_{n}=\infty$ a.s. However, $\lim n^{-1} S_{n}=\infty$ a.s. certainly implies that $\lim \sup T_{n}=-\infty$ a.s. Thus (b) holds.

Conversely, suppose that (b) holds. Then, for each $a>0$,

$$
\sum n^{-1} P\left(S_{n}-n a<0\right)<\infty
$$

${ }^{1}$ Partially supported by National Science Foundation (grant GP-5217). 
and consequently, from the same work of Spitzer, we have that

$$
\max _{k \geq 1}\left(k a-S_{k}\right)+<\infty \text { a.s. }
$$

for all $a>0$. This clearly implies that $\lim n^{-1} S_{n}=\infty$ a.s., and therefore (a) holds.

Before proceeding to the proof of the theorem, we note that previous work (e.g. Derman and Robbins [1]) giving sufficient conditions that $\lim n^{-1} S_{n}=\infty$ a.s. follows quickly from the above lemma.

Proof of the Theorem. Let $\left\{Y_{k}\right\}$ denote a sequence of i.i.d. random variables, each with characteristic function $\left(1+u^{2}\right)^{-1}$, and, further, let $\left\{Y_{k}\right\}$ be independent of $\left\{X_{k}\right\}$. Write $Z_{k}=X_{k}+Y_{k}$ and

$$
W_{n}=\sum_{k=1}^{n} Z_{k} \quad(n=1,2, \cdots) .
$$

Since $Y_{k}$ has expectation zero, $n^{-1} S_{n} \rightarrow \infty$ a.s. if and only if $n^{-1} W_{n}$ $\rightarrow \infty$ a.s.

By means of a well-known inversion formula, we have that

$$
P\left(a n-b \leqq W_{n}<a n\right)=\frac{1}{2 \pi} \int_{-\infty}^{\infty} e^{-i u a n}\left(\frac{e^{i u b}-1}{i u}\right)\left(\frac{\phi(u)}{1+u^{2}}\right)^{n} d u .
$$

(Note that $\left\{\phi(u) /\left(1+u^{2}\right)\right\}^{n}$ is integrable and that $W_{n}$ has an absolutely continuous distribution function.)

Therefore,

$$
\begin{aligned}
\sum_{n=1}^{\infty} n^{-1} P\left(a n-b \leqq W_{n}<\right. & a n) \\
& =\frac{1}{2 \pi} \int_{-\infty}^{\infty} \frac{e^{i u b}-1}{i u} \log \left\{1-\frac{e^{-i u a} \phi(u)}{1+u^{2}}\right\}^{-1} d u,
\end{aligned}
$$

the interchange of sum and integral being justified, since

$$
\begin{aligned}
& \int_{-\infty}^{\infty} \sum_{n=1}^{\infty}\left|\frac{1}{n} e^{-i u a n}\left(\frac{e^{i u b}-1}{i u}\right)\left(\frac{\phi(u)}{1+u^{2}}\right)^{n}\right| d u \\
& \leqq \vdots \int_{-\infty}^{\infty}\left\{\sum_{n=1}^{\infty} \frac{1}{n}\left|\frac{\phi(u)}{1+u^{2}}\right|^{n}\right\} d u \\
& \leqq b \int_{-\infty}^{\infty}\left\{\sum_{n=1}^{\infty} \frac{1}{n}\left(\frac{1}{1+u^{2}}\right)^{n}\right\} d u \\
& \quad=b \int_{-\infty}^{\infty} \log \left(1+\frac{1}{u^{2}}\right) d u \\
& \quad<\infty .
\end{aligned}
$$


From the Monotone Convergence Theorem, it follows that

$$
\begin{aligned}
\sum_{n=1}^{\infty} n^{-1} P\left(W_{n}<a n\right) & =\lim _{b \rightarrow \infty} \sum_{n=1}^{\infty} n^{-1} P\left(a n-b \leqq W_{n}<a n\right) \\
& =\lim _{b \rightarrow \infty} \int_{-\infty}^{\infty} \frac{e^{i u b}-1}{i u} \log \left(1-\frac{e^{-i u a} \phi(u)}{1+u^{2}}\right)^{-1} d u .
\end{aligned}
$$

By the Riemann-Lebesgue lemma,

$$
\lim _{b \rightarrow \infty} \int_{|u|>1} \frac{e^{i u b}-1}{i u} \log \left(1-\frac{e^{-i u a} \phi(u)}{1+u^{2}}\right)^{-1} d u
$$

exists and is finite. It follows that (1) is finite for each $a>0$ if and only if $\sum n^{-1} P\left(W_{n}<a n\right)$ is finite for each $a>0$. The latter condition is equivalent to $n^{-1} W_{n} \rightarrow \infty$ a.s. which is, in turn, equivalent to $n^{-1} S_{n} \rightarrow \infty$ a.s. This completes the proof.

\section{REFERENCES}

1. C. Derman and H. Robbins, The strong law of large numbers when the first moment does not exist, Proc. Nat. Acad. Sci. U.S.A. 41 (1955), 586-587.

2. F. Spitzer, A combinatorial lemma and its application to probability theory, Trans. Amer. Math. Soc. 82 (1956), 323-339.

\section{Royal Holloway College, London University and}

State University of New York at Albany 